\title{
Cognitive Aging and the Hippocampus: How Old Rats Represent New Environments
}

\author{
Iain A. Wilson, ${ }^{1}$ Sami Ikonen, ${ }^{1}$ Irina Gureviciene, ${ }^{1}$ Robert W. McMahan, ${ }^{2}$ Michela Gallagher,${ }^{2}$ Howard Eichenbaum, ${ }^{3}$ \\ and Heikki Tanila ${ }^{1,4}$ \\ ${ }^{1}$ Department of Neuroscience and Neurology, University of Kuopio, Kuopio 70211, Finland, ${ }^{2}$ Department of Psychology, Johns Hopkins University, \\ Baltimore, Maryland 21218, ${ }^{3}$ Department of Psychology, Boston University, Boston, Massachusetts 02215, and ${ }^{4}$ Department of Neurology, Kuopio \\ University Hospital, Kuopio 70211 Finland
}

Spatial learning impairment in aged rats is associated with changes in hippocampal connectivity and plasticity. Several studies have explored the age-related deficit in spatial information processing by recording the location-specific activity of hippocampal neurons (place cells). However, these studies have generated disparate characterizations of place cells in aged rats as unstable (Barnes et al., 1997), resistant to change (Tanila et al., 1997b; Oler and Markus, 2000; Wilson et al., 2003), or delayed in using external cues (Rosenzweig et al., 2003). To reconcile these findings, we recorded place cells from aged and young rats as they repeatedly explored both a highly familiar environment and an initially novel environment, and we repeatedly tested whether the place fields formed in the novel environment were anchored by external cues. Initially, spatial representations in aged rats were abnormally maintained between the familiar and novel environments. Then, new representations were formed but were also delayed in becoming anchored to the external landmarks. Finally, even when the new spatial representations became bound to the landmarks, they were multi-stable across repetitive exposures to the formerly novel environment. These observations help to reconcile previously divergent characterizations of spatial representation in aged rats and suggest a model of cognitive aging and hippocampal function.

Key words: place cells; spatial memory; aging; hippocampus; novel environment; age-associated cognitive impairment

\section{Introduction}

Hippocampal CA1 and CA3 pyramidal cells are highly active when a rat occupies particular places within an environment, and these "place cells" serve as a prominent reflection of hippocampal spatial information processing (O'Keefe and Dostrovsky, 1971). Aging in humans and animals is accompanied by a decline in learning capacity, particularly in spatial memory (for review, see Barnes, 1988; Rapp and Amaral, 1992) (Uttl and Graf, 1993; Mizumori and Kalyani, 1997). Furthermore, this cognitive impairment is associated with deterioration of hippocampal circuitry and plasticity (Fischer et al., 1992; Barnes, 1994; Smith et al., 2000), suggesting that the ageassociated spatial learning impairment might be explained by deficiencies in hippocampal spatial information processing.

Several studies have examined place cells in aged rats and have generated divergent views on the nature of age-associated changes in spatial representation. Tanila and colleagues (Wilson et al., 2003) described place cells in aged rats as "rigid," in that they maintain the same spatial representation abnormally across multiple environments that share some cues. In contrast, Barnes et al. (1997) characterized place cells in aged rats as "multi-stable,"

Received Nov. 1, 2003; revised March 2, 2004; accepted March 2, 2004.

This work was supported by National Institute on Aging Grant AG09973 and Academy of Finland Grant 46000. We thank Dr. Matthew Shapiro for much of the analysis software and Kestutis Gurevicius for several analysis programs.

Correspondence should be addressed to lain A. Wilson, Department of Neuroscience and Neurology, University of Kuopio, P.0. Box 1627, Harjulantie 1, Kuopio 70211, Finland. E-mail: iain.wilson@uku.fi.

D0I:10.1523/JNEUROSCI.5205-03.2004

Copyright $\odot 2004$ Society for Neuroscience $\quad$ 0270-6474/04/243870-09\$15.00/0 that is, unpredictably switching among multiple spatial firing patterns on repeated explorations of the same environment. Alternatively, Rosenzweig et al. (2003) have characterized place cells in aged rats as delayed in anchoring spatial representations to salient external cues. These characterizations are at least superficially contradictory, differently suggesting that place cells in aged rats are, respectively, overly stable, unstable, or delayed in stabilizing to external stimuli.

The discrepancies in these characterizations may arise from differences in the experimental protocols used to explore the nature of spatial representations (Rapp, 1998; Redish et al., 1998; Wood and Dudchenko, 2003). Barnes et al. (1997) recorded rats in a familiar environment before and after an exposure to a novel area. Tanila and colleagues, as well as Oler and Markus, compared variations of an environment that intentionally shared many spatial cues (Tanila et al., 1997b; Oler and Markus, 2000; Wilson et al., 2003). Rosenzweig et al. (2003) manipulated a single environment to put self-motion and external cues into conflict with one another. To investigate the relationships between the previously described properties of aged place cells, we recorded from aged and young rats as they repeatedly explored both a highly familiar environment and an initially novel environment that differed in both geometry and all the salient spatial cues from the familiar environment, and we repeatedly tested whether the place fields in the new environment were anchored to external visual cues by occasionally rotating the arena frame. Under these conditions, we observed a course of place cell firing patterns that sequentially reflected each of the previous descriptions of age-associated abnormalities in spatial representation. 


\section{Materials and Methods}

Subjects and surgery

The subjects were eight young (6-8 months) and 11 aged (24-26 months) Long-Evans male rats (experiment $1, n=6$ young rats, $n=9$ aged rats; experiment $2, n=2$ young rats, $n=2$ aged rats). These rats were prescreened for performance in the Morris water maze at Johns Hopkins University. After this testing, the rats were shipped to the University of Kuopio for electrode implantation and recordings. Under anesthesia (pentobarbital and chloral hydrate each at $40 \mathrm{mg} / \mathrm{kg}$, i.p., supplemented with ketamine at $20 \mathrm{mg} / \mathrm{kg}$ i.m.), each rat was implanted with two movable tetrodes (a bundle of four twisted $30 \mu \mathrm{m}$ Nichrome wires) aimed at CA1 of each hemisphere (anteroposterior, -3.3; mediolateral, +1.8 ; dorsoventral, -2.2 from the dura). In addition, bipolar stimulation electrodes were implanted in the lateral hypothalamus to deliver rewarding brain stimulation. The stimulus current was adjusted to the minimum level that kept each rat constantly moving. Additional details of the recording and stimulation techniques have been described previously (Ikonen et al., 2002), and the implant construction and the surgical procedure have also been described previously (Tanila et al., 1997a). All animal experiments were conducted in accordance with National Institutes of Health and Council of Europe guidelines.

\section{Behavioral protocols}

Water maze. Rats were trained on a spatial navigation task in the Morris water maze using training trials to assess the course of acquisition and probe trials to assess search strategies in locating a submerged escape platform. Rats received three trials per day for 8 consecutive days using a $60 \mathrm{sec}$ intertrial interval. The location of the platform remained constant in one quadrant of the maze, and the starting position for each trial was varied among four equally spaced positions around the perimeter of the maze. Every sixth trial was a probe trial, during which the platform was retracted and unavailable for escape for the first $30 \mathrm{sec}$ of the trial; after this time, it was raised and made available for escape. The probe trials assessed whether there was development of a spatial bias in searching for the escape platform. To test visual acuity and swimming ability independent of the ability to process spatial information, each rat was given six "cued" training trials on the day after completion of the spatial training trials. During these trials, the submerged platform was replaced with a visible platform at $2 \mathrm{~cm}$ above the surface of the water, and the location of the escape platform was varied randomly among the quadrants of the pool from trial to trial. Each rat was allowed $30 \mathrm{sec}$ to reach the platform and was allowed to remain there briefly before being returned to a holding cage for $5 \mathrm{sec}$ before the next trial. The behavioral protocol and data analysis have been described in detail previously (Gallagher and Burwell, 1989). The primary measure, referred to as the learning index, is derived from the probe trials that were interpolated after each set of five training trials. The learning index is computed as the average proximity (in centimeters) of the rat to the target platform location on probe trials two to four; thus, low index values represent more accurate search patterns acquired more rapidly during learning, whereas high index values indicate an inaccurate search strategy.

Place cell recordings. The familiar environment was a brown pasteboard cylinder $(70 \mathrm{~cm}$ diameter, $50 \mathrm{~cm}$ height), with three distinct twodimensional patterns attached to the cylinder wall serving as landmark cues. Within this environment, the animals were trained to search for randomly distributed loci in which rewarding brain stimulation was delivered. The rats became highly familiar with this environment during the

Experiment 1, session 2

Firing rate (spikes/sec)

Fam1

Spatial selectivity

Slope (weighted)

Fam1

New1

Information content

Fam1

Running speed $(\mathrm{cm} / \mathrm{sec})$ Fam1

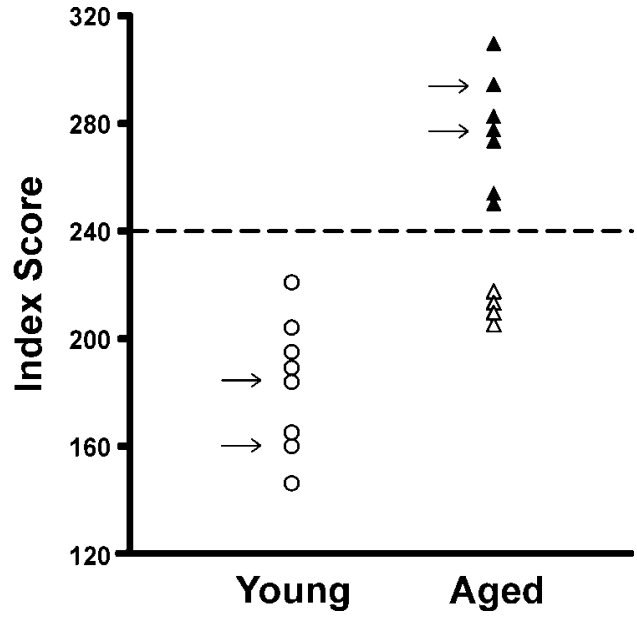

Figure 1. Learning index scores from the behavioral characterization of the individual rats used in the recording studies. The arrows indicate the subjects used in experiment 2 . The learning index is derived from interpolated probe trials during place learning in the water maze (Gallagher et al., 1993). Lower values represent more rapid and accurate acquisition of a search for the escape platform.

Table 1. Basic place cell and behavioral characteristics in young versus aged rats

\begin{tabular}{lcll}
\hline & Young (mean \pm SEM) & Aged (mean \pm SEM) & Significance of age \\
\hline $\begin{array}{c}\text { Experiment 1, session 1 } \\
\text { Firing rate (spikes/sec) }\end{array}$ & & \\
$\quad$ Fam1 & $0.35 \pm 0.06$ & $1.06 \pm 0.29$ & $t_{(13)}=-2.4 ; p=0.04$ \\
$\quad$ New1 & $0.56 \pm 0.18$ & $1.34 \pm 0.28$ & $t_{(13)}=-2.0 ; p=0.05$ \\
Spatial selectivity & & \\
Slope (weighted) & & & NS \\
Fam1 & $-0.05 \pm 0.02$ & $-0.07 \pm 0.01$ & NS \\
New1 & $-0.06 \pm 0.01$ & $-0.06 \pm 0.01$ & NS \\
Information content & & & NS \\
Fam1 & $1.9 \pm 0.2$ & $1.6 \pm 0.2$ & \\
New1 & $1.8 \pm 0.4$ & $1.4 \pm 0.2$ & NS \\
Running speed (cm/sec) & & & NS \\
Fam1 & $17.4 \pm 2.0$ & $14.2 \pm 1.6$ & $13.4 \pm 1.1$ \\
New1 & $14.4 \pm 0.5$ & & \\
\hline
\end{tabular}

$0.51 \pm 0.16$

$0.82 \pm 0.17$

$0.95 \pm 0.19$

NS

$0.48 \pm 0.23$

$-0.07 \pm 0.01$

$-0.09 \pm 0.04$

$-0.12 \pm 0.03$

$0.0 \pm 0.06$

NS

$1.8 \pm 0.1$

$3.0 \pm 0.9$

$1.4 \pm 0.2$

$1.7 \pm 0.2$

NS

$21.0 \pm 2.5$

$16.3 \pm 1.8$

$17.7 \pm 2.2$

$13.6 \pm 1.2$

NS

NS
2-3 weeks of daily screening for cells. During each recording session, the rats were initially exposed to the familiar cylindrical environment (Fam), and then they were introduced to a novel environment (New) for multiple trials and were returned to the familiar environment in a final trial. The novel environment was formed by black plastic walls composed into a hexagon $(80 \mathrm{~cm}$ diameter, $40 \mathrm{~cm}$ height), and a novel and distinct landmark cue was positioned on each of three walls. Both arenas were frames placed on the same black plastic table ( $1.1 \mathrm{~m}$ diameter) that was cleaned thoroughly between trials to minimize olfactory cues. The table 
and frames were surrounded by a floor-toceiling circle of black curtain ( $2.2 \mathrm{~m}$ diameter); the rat remained inside this curtained area for the entirety of each recording session. The arena itself was lit by four incandescent bulbs arranged symmetrically $1.5 \mathrm{~m}$ above the center of the arena floor. The room was almost soundproof, with the ventilation creating a diffuse background white noise. These factors all served to limit the orienting cues outside the arena frame.

In experiment 1 , each initial recording session consisted of five 7 min trials (Faml-New1New2-New3-Fam2) separated by 5 min pauses, during which the rat was placed in a bucket (40 $\mathrm{cm}$ diameter, $80 \mathrm{~cm}$ height) suspended above the arena while environment manipulations were made. The bucket was spun to mildly disorient the animals before reentry, with the exception of between trial New1 and trial New2. The rats underwent two recording sessions separated by 3-4 d. The second session allowed us to test for delayed acquisition of spatial representations for the novel environment. In addition, in one of the exposures to the novel environment, we tested whether the spatial representations were anchored to the landmark cues by rotating those cues and the arena frame by $90^{\circ}$. Hence, session 2 followed the sequence Fam1-New1-New2New3R-Fam2.

In experiment 2, we recorded place cells from an additional two aged memory-impaired (28 months old) and two young (8 months old) rats given repeated exposures to the novel environment. Each rat explored the same familiar and novel arenas for as many as eight consecutive daily sessions with the sequence Fam1New1-New2R-New3-Fam2. Trial New2R was a $90^{\circ}$ rotation of the entire arena including landmarks. For counterbalancing, the direction of rotation alternated each session, either $90^{\circ}$ clockwise or counterclockwise.

\section{Spatial firing pattern analysis}

The recordings were made with Enhanced Dis-

covery software, and cells were isolated off-line by clusters defined with waveform parameters using Autocut software (both from DataWave Technologies, Longmont, CO). Place cells were defined as pyramidal cells (on the basis of the presence of complex spikes and duration of the negative spike $>300 \mu \mathrm{sec}$ ) with a place field in at least one of the recording environments. Place fields were defined as a set of at least six adjacent pixels with firing rates above $0.5 \mathrm{~Hz}$ and two times above the overall mean firing rate of the cell. To compare the properties of place cells between groups, all analyses were based on the firing rates, which were calculated for periods when the rat was moving at least $2 \mathrm{~cm} / \mathrm{sec}$. We also compared spatial selectivity, calculated in two ways: first, by the spatial information content (Skaggs et al., 1993; Markus et al., 1994), and second, by the weighted slope of the first-order to fourth-order autocorrelations (a measure of the place field profile). These autocorrelations correspond to four concentric rings around each arena pixel $(3.5 \times 3.5 \mathrm{~cm})$ and describe the field profile of all pixels with a non-zero firing rate. The slope of these autocorrelations quantifies the change in the field profile as one moves farther from the central pixel; a steep slope indicates a high spatial selectivity. The slope was weighted to give greatest influence to the first autocorrelations. The weighted slope was calculated as follows: $\left[(\mathrm{ac} 1-\mathrm{ac} 2)^{\star} 3+(\mathrm{ac} 2-\mathrm{ac} 3)^{\star} 2+(\mathrm{ac} 3-\mathrm{ac} 4)\right] / 6$, where ac1 is firstorder autocorrelation, ac2 is second-order correlation, etc. (for additional details, see Wilson et al., 2003).

To measure the extent to which a spatial representation changed be-
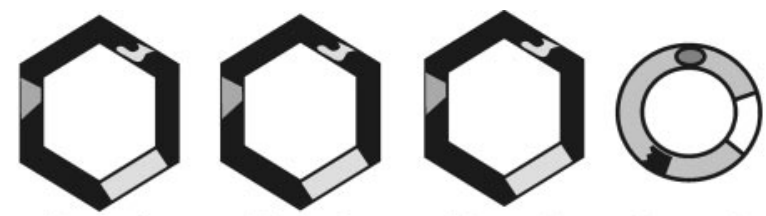

New2

New3

Fam2

\section{A. Young}
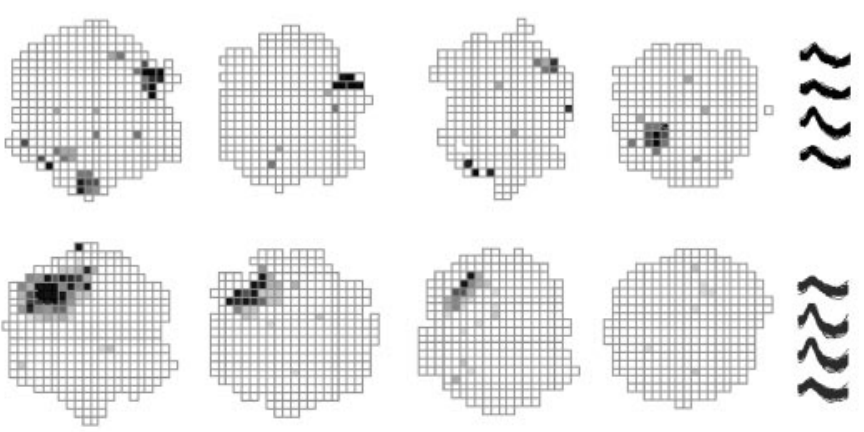

\section{B. Aged}

Figure 2. Session 1: initial spatial representations of a novel environment. Place fields of hippocampal cells in rats exploring the familiar cylinder (Fam) and the novel hexagon (New) arenas during session 1 are shown. Data are shown for two cells of two young (rat $\mathrm{H} 5$ cell 4 and rat $\mathrm{G} 9$ cell 12) ( $A$ ) and for two cells of two aged rats (rat $\mathrm{G} 6$ cell 8 and rat $\mathrm{H} 2$ cell 3 ) (B). In session 1, the field changes in the surrounding environments. Firing rate scales in spikes per second are provided on the left; example waveforms

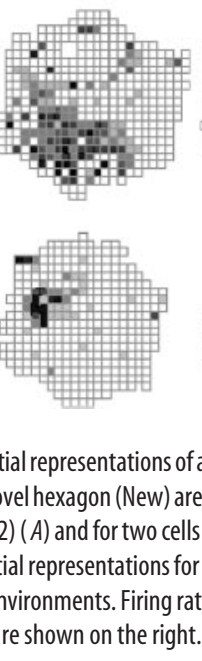

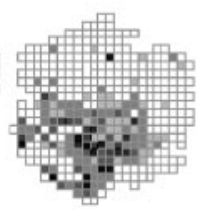
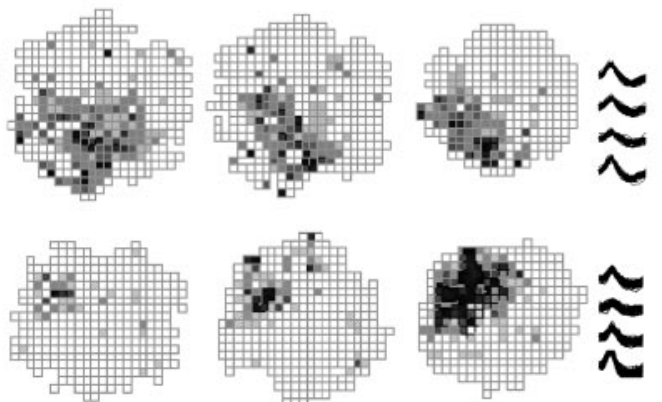

tween manipulations of the environment, we quantified the similarity between firing rate maps calculated as pixel-to-pixel $(3.5 \times 3.5 \mathrm{~cm})$ cross-correlations. The slightly larger hexagon maps were first scaled down to the cylinder size. For each environment, the spatial selectivity and pixel-to-pixel correlation were calculated only if the mean firing rate of the cell was above $0.1 \mathrm{~Hz}$. In addition, for analysis of the rotated novel environment (experiment 1, session 2, New3R), we selected only those cells that had place fields in the novel environment before the rotation (New2). Because of this restriction, one young rat that had no place fields in the novel environment was not included in this session 2 analysis. We examined the similarity of spatial representations between the New and rotated-New trials by comparing trial New 2 and the trial New3R rotated by $\pm 90^{\circ}$ to bring the landmarks into alignment (New3RA). As a second way of measuring the response to the change in environment, we measured the magnitude of firing rate change (independent of direction) across environments, calculated as follows: ABS [(fr1 - fr2 $) /(\mathrm{fr} 1 \pm \mathrm{fr} 2)]$ $\times 100 \%$, such that $\mathrm{ABS}$ is absolute value, and fr is firing rate. This measure allowed us to account for cells that were silent each time in an environment, an occasion when the field correlations could not be used. Finally, we measured the running speed of the rat to control for immobility periods.

\section{Histology}

At the end of the study, the rats were deeply anesthetized, and the recording sites were marked by passing anodal current $(30 \mu \mathrm{A}, 5 \mathrm{sec})$ through 
the electrodes. The animals were perfused with buffered $4 \%$ Formalin, and the brains were cut into $50 \mu \mathrm{m}$ sections. The locations of the electrodes tips were confirmed by Prussian blue reaction, as described previously (Tanila et al., 1997a).

\section{Results \\ Experiment 1}

Spatial learning

As found in previous studies (Gallagher et al., 1993), the aged (24-26 months) rats as a group had a significantly higher spatial learning index, indicating impaired spatial memory, compared with young adult $(6-8$ months $)$ rats $(245 \pm 12 \mathrm{vs} 187 \pm 11 \mathrm{~cm}$, mean $\left.\pm \operatorname{SEM} ; t_{(13)}=3.3 ; p=0.006\right)$. As shown in Figure 1 , some of the aged rats had scores within the range of young performance, whereas others performed outside the normative range ( $>240$ index score) of the young subject population (Gallagher et al., 1993). In contrast, during simple training to locate a visible platform, the aged rats had escape latencies equivalent to those of young rats $\left(7.3 \pm 1.2 \mathrm{vs} 8.1 \pm 1.4 \mathrm{sec}\right.$, respectively; $t_{(13)}=0.43$; $p>0.60)$.

Place cells

Subsequently, we recorded simultaneously from multiple (4.9 \pm 0.5 , mean \pm SEM) hippocampal place cells as the rats searched randomly for loci associated with rewarding brain stimulation in the familiar and altered arenas. Twenty-one CA1 and 25 CA3 place cells were recorded from the young rats, and 65 CA1 and 36 CA3 place cells were recorded from the aged rats. Because there were no differences between CA1 and CA3 cells in any of the above described measures, the data from the two hippocampal areas were pooled.

\section{Spatial representations in the familiar environment}

Our analyses focused on three parameters that characterize the physiology of place cells and behavior during exploration of an environment: the overall mean firing rate, spatial selectivity, and spatial stability. In the familiar environment (Fam1), the basic properties of place cells and the general behavior of the aged and young rats were similar, although the place cells of aged rats did have higher average firing rates than those of the young rats (in both CA1 and CA3) (for details, see Table 1, top). In both young and aged animals, the spatial representations were stable across repetitions of exposures to the familiar environment (correlation Fam1-Fam2: young, $0.21 \pm 0.06$; aged, $0.30 \pm 0.03 ; t_{(13)}=-1.0$; $p>0.1)$.

\section{Representation of a novel environment}

The overall firing rates of hippocampal cells continued to be significantly higher among the aged rats (Table 1, top). Furthermore, mean firing rates (the population mean of all simultaneously recorded cells) increased for both groups during introduction to the novel environment (environment, $F_{(1,13)}=9.7$; $p=0.008)$, and the increase was not greater in young rats than aged rats (environment $\times$ age interaction, $F_{(1,13)}=0.2 ; p>0.5$ ). However, an additional analysis showed that average absolute values of firing rate changes for all cells in each rat were greater in young than aged rats (firing rate percentage change: young, $42 \pm$ $2 \%$; aged, $\left.26 \pm 6 \% ; t_{(13)}=2.4 ; p=0.03\right)$.

Despite the differences in all of the salient external cues and shape of the novel environment, the pixel-by-pixel correlation analysis of spatial representations revealed that the spatial representations of some aged animals persisted from the familiar environment during the initial exposure to the novel environment. In contrast, hippocampal cells of young rats developed new spatial representations during the first trial (correlation Fam1-New1: young, $-0.03 \pm 0.03$; aged, $0.14 \pm 0.07 ; t_{(13)}=-2.3 ; p=0.04$ ). Figure 2 provides examples from hippocampal ensembles of spatial representations in trials Fam 1 and New1, showing the rapid generation of new spatial representations in young rats, in contrast to the rigidity of hippocampal spatial representations in aged rats after introduction to the novel arena. Table 2 provides the percentage of cells within each animal that had a high correlation between Fam1 and New1. There was no significant correlation between the spatial learning index and the place field similarity between the environments (Spearman's $r_{(15)}=0.2 ; p>0.1$ ).

In addition, we examined the stability of spatial representations associated with the novel environment (for the percentages of cells with high New1-New2 correlations, see Table 2). The place fields of the aged animals were significantly more stable

Table 2. Place field correlations in cell ensembles of individual rats

\begin{tabular}{|c|c|c|c|c|c|c|c|}
\hline \multirow[b]{2}{*}{ Rat } & \multirow[b]{2}{*}{ Memory index } & \multicolumn{3}{|c|}{ Session 1} & \multicolumn{3}{|c|}{ Session 2} \\
\hline & & Cells S1 & Fam1-New1 (same \%) & New1-New2 (same \%) & Cells S2 & Fam1-New1 (same \%) & New2-New3RA (rot \%) \\
\hline \multicolumn{8}{|l|}{ Young } \\
\hline H8 & 164 & 7 & 14 & 100 & 3 & 33 & 100 \\
\hline $\mathrm{H} 10$ & 189 & 3 & 0 & 67 & 0 & No fields & No fields \\
\hline G9 & 195 & 6 & 0 & 50 & 10 & 0 & 90 \\
\hline G7 & 204 & 1 & 0 & 0 & 3 & 33 & 100 \\
\hline \multicolumn{8}{|l|}{ Aged } \\
\hline H1 & 204 & 8 & 13 & 100 & 4 & 0 & 100 \\
\hline $\mathrm{H} 2$ & 211 & 6 & 83 & 83 & 4 & 75 & 67 \\
\hline G3 & 213 & 8 & 0 & 63 & 7 & 0 & 100 \\
\hline $\mathrm{H} 3$ & 215 & 2 & 50 & 100 & 2 & 0 & 0 \\
\hline H6 & 250 & 1 & 100 & 100 & 1 & 0 & 0 \\
\hline Aged mean & 245 & 5.8 & $32 \%$ & $84 \%$ & 5.2 & $13 \%$ & $38 \%$ \\
\hline
\end{tabular}

Cells were determined to have similar firing field patterns across two trials by meeting a criterion level of 0.25 cross-trial correlation, and the percentage of these cells for each rat shows the coherence of ensembles within and across rats. Zero and $100 \%$ indicate coherent cell ensembles, whereas a response of $50 \%$ represents maximum discordance within the cell population. The rotated trial New3RA was aligned so correlations above 0.25 represent cells that did rotate with the landmarks. 
than those of the young animals (correlation New1-New2: young, $0.27 \pm 0.04$; aged, $\left.0.41 \pm 0.03 ; t_{(12)}=-3.0 ; p=0.01\right)$. It is likely that this arises from the lack of development of new spatial representations for the novel environment by the aged rats.

These results gave us the opportunity to explore further the failure of hippocampal cells in aged rats to distinguish between two very different environments. On a second day of exposure to the novel environment, we looked at the role of the amount of experience and importance of the visual landmarks on the encoding of two distinct arenas.

\section{Session 2: the second exposure to the} novel environment

In the second recording session performed 3-4 d later, the basic cellular firing characteristics and general behavior of the young and aged rats were little changed from the first session (Table 1, bottom). However, in contrast to the first session in the novel arena, on the second day of exposure both the young and aged rats treated the hexagon environment as different from the familiar cylinder. Now the average absolute firing rate change was similar for cells of both groups of animals (young, $37 \pm 11 \%$; aged, $25 \pm 6 \%$; $t_{(12)}=$ $1.0 ; p>0.30)$. Also, during initial exposure to the novel environment in the second session, the place cells of aged animals developed distinct spatial representations, much as the young rats did during their first exposure on the first day of recording (Fig. 3). In trial New1, neither group showed a large amount of carry over of place field representations from the familiar to the novel environment (correlation Fam1-New1: young, $0.03 \pm 0.04$; aged, $0.05 \pm 0.04 ; t_{(12)}=$ $-0.4 ; p>0.50$ ) (Table 2). Additionally, the stability within the hexagon arena was not different between the groups for the second session (correlation New1-New2: young, $0.43 \pm 0.05$; aged, $0.46 \pm$ $\left.0.03 ; t_{(11)}=-0.6 ; p>0.50\right)$. These data suggest that the aged animals required more time or experience than young rats to encode the change in environment.

We further tested how well the visual landmarks were incorporated into the new spatial representation by rotating the arena $90^{\circ}$ clockwise in trial New3R. As Figure 3 illustrates, the place fields of young rats clearly rotated $90^{\circ}$ clockwise, whereas the spatial representations of some aged rats failed to follow the visual cues. We analyzed the place field rotations by aligning the rotation firing rate maps to the originals (New3RA, $90^{\circ}$ counterclockwise alignment rotation). Because cells of some of the aged animals followed the cues whereas others did not (as shown by Table 2), the means of the young and aged rats were not significantly different (New2-New3RA correlation: young, $0.29 \pm 0.08$; aged, $\left.0.22 \pm 0.09 ; t_{(12)}=0.6 ; p>0.5\right)$. During additional analysis, we found that those rats whose spatial firing patterns failed to rotate with the landmarks were also the rats that performed most the right.
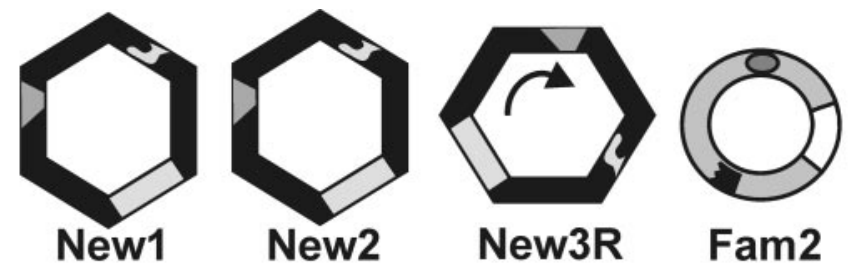

(Rot $\left.-90^{\circ}\right)$
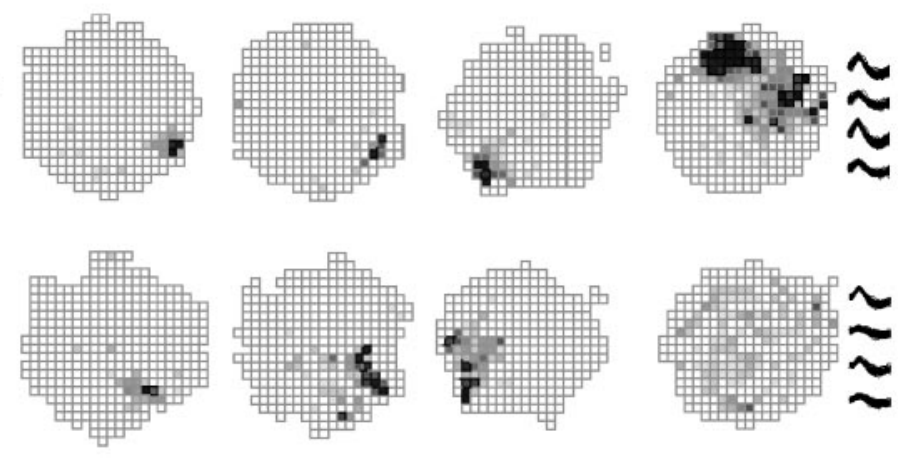

Figure 3. Session 2: spatial representations in the second exposure to the novel arena. Place fields of hippocampal cells in rats exploring the familiar cylinder (Fam) and the novel hexagon (New) arenas during session 2 are shown. Data are shown for two cells the fields of young and aged rats formed distinct spatial representations for each environment. When the new hexagon arena of trial New2. Firing rate scales in spikes per second are provided on the left; example tetrode waveforms of each cell are shown on

poorly on the water maze test. Conversely, the rats whose place fields rotated with the cues performed best on the water maze (all subjects: Spearman's $r_{(14)}=-0.61, p=0.02$; young only: $r_{(5)}=0.1$, $p>0.8$; aged only: $\left.r_{(9)}=-0.68, p=0.04\right)$. Figure 4 depicts the correlation between memory index and place field rotations. In contrast, rats whose place fields rotated with the landmarks did not perform any better on the visible platform water maze test than those whose fields did not rotate (Spearman's $r_{(14)}=-0.3 ; p>0.20$ ).

The lack of rotation among the aged memory-impaired animals suggests that they either do not attend to the visual landmarks or, in concordance with theories proposed by McNaughton et al. (1996), the aged rats require additional experience for the hippocampal representation to be bound to the visual landmarks by associative learning. To assess whether landmark encoding would become consistent with greater experience, we used four additional rats (two young and two aged memoryimpaired) in a second experiment, providing up to eight rotation sessions with the novel environment. 


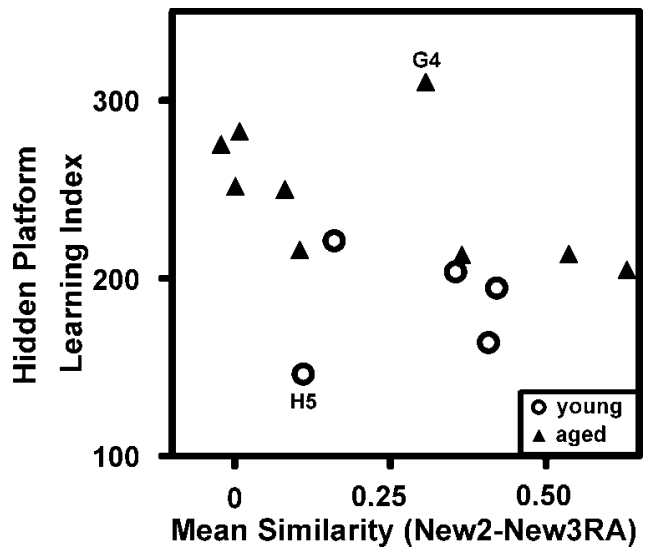

Figure 4. High correlation between learning index and place field rotations with novel cues. The spatial learning index correlated well with the similarity of spatial representations between the trials New2 and New3RA with the firing rate map of New3R rotated by $+90^{\circ}$ to bring the landmarks into alignment (hence, the code New3RA, for New3 rotation-aligned). Poor spatial memory is indicated by high learning index scores and is linked here to failures of spatial representations to follow landmarks. High similarity scores (rotation with the landmarks) correlated positively with spatial memory performance. The statistics are as follows: for all subjects, Spearman's $r_{(14)}=-0.61, p=0.02$; for young only, $r_{(5)}=0.1, p>0.8$; for aged only, $\left.r_{(9)}=-0.68, p=0.04\right)$. Regarding the two outliers, the young rat $\mathrm{H} 5$ had one cell that clearly rotated and one cell that ceased firing. The aged rat G4 had five cells: three of them rotated, whereas two fired associated with both the original and rotated positions.

\section{Experiment 2}

The two aged memory-impaired rats ( 28 months old) performed poorly in the water maze test of spatial navigation with index scores of 277 and 294, whereas the young rats (8 months old) achieved index scores of 186 and 160 . We recorded from multiple $(4.0 \pm 0.5)$ place cells as the rats explored the novel arena. From the aged rats, we recorded $60 \mathrm{CA} 1$ and eight CA3 place cells over eight sessions per animal. From the young rats, we recorded 34 CA1 place cells over six and three sessions per animal. Neurons from the CA1 and CA3 layers were pooled.

During the initial session, place cells of the two aged memoryimpaired rats again failed to rotate with the landmarks in the new environment, whereas the place cells of the two young rats clearly did rotate (rotation-aligned correlations: young, $0.32 \pm 0.06$; aged, $-0.04 \pm 0.04$ ). Figures 5 and 6 illustrate sample cells from ensembles recorded from each session for each rat. With additional exposures to the hexagon arena and its rotation, the place cells of aged rats followed the landmarks during the second (rat R5) or third (rat R8) session, as determined by an aligned correlation score of $>0.25$ (correlations similar to young rats). This suggested that the spatial representations of the aged rats had become bound to the environmental landmarks. In subsequent sessions, however, the place cells of aged rats did not consistently follow the landmark rotations, as evidenced by aligned correlations of $\sim 0$ (for mean aligned correlations of each neuronal ensemble recorded in all four rats over all sessions, see Fig. 7). Moreover, in the seventh and eighth sessions for aged rats, the cells even produced two representations of the same environment showing another variety of fluctuation (Fig. 6). Thus, during repeated exposures to the novel environment, the place fields of aged memory-impaired rats did not rotate with the landmarks initially but thereafter fluctuated with rotation on some but not all occasions, whereas the place fields of young rats always rotated with the landmarks.

\section{Discussion}

Previous studies have reported that aging results in weakened control of external cues over hippocampal spatial representations. How- ever, different experiments have described different manifestations of this weakened control (Barnes et al., 1997; Tanila et al., 1997b; Oler and Markus, 2000; Rosenzweig et al., 2003; Wilson et al., 2003). This study takes a significant step toward reconciling these divergent characterizations by observing that place representations of hippocampal cells in aged rats are rigid, delayed in acquiring control by external cues, and multi-stable at different stages of spatial experience in the same experiment. Our observation that these manifestations of aging occur sequentially suggests a model of hippocampal aging that can be related to neurobiological changes in hippocampal circuitry during aging.

Rigidity, delayed control by external cues, and multi-stability When moved from a familiar environment to a novel one, hippocampal neurons of young rats rapidly develop spatial representations that are unrelated to those of the familiar environment, and these representations are strongly under the control of salient visual landmarks (Wilson and McNaughton, 1993; Shapiro, 2001). Previous experiments have demonstrated that hippocampal cells of aged rats abnormally maintain existing spatial representations over multiple experiences despite alterations in environment or task demands sufficient to induce new representations in young rats during the first experience (Tanila et al., 1997b; Oler and Markus, 2000; Wilson et al., 2003). The current experiment demonstrates that hippocampal spatial representations in aged rats abnormally persist even into an environment in which all salient spatial cues are novel and only subtle internal or external cues are maintained. However, under the current conditions, the rigidity between two distinct environments is temporary. Also, consistent with findings by Rosenzweig et al. (2003), place fields of aged rats in the present study were not initially controlled by the external cues. With additional experience, place fields of aged rats did rotate with the landmarks, demonstrating that the hippocampal spatial representations in aged rats were eventually bound to external cues. However, even after numerous repeated exposures, the hippocampal spatial representations of aged memory-impaired rats rotated with the landmarks on some but not all occasions and differed across experiences within the same environment, reflecting the characteristics of "multi-stability" described by Barnes et al. (1997).

\section{Sources of weakened external cue control}

In our view, there are four possible explanations for weakened control of external cues over hippocampal spatial representations as aged rats learn a new environment. First, aged rats may have increased emphasis on self-motion information instead of external cue information. Idiothetic cues, as well as external spatial cues, contribute strongly to hippocampal spatial representations (McNaughton et al., 1996) and participate in navigation through separate, competing learning systems (White and McDonald, 2002). In behavioral tests using a T maze, Barnes et al. (1980) found that aged rats rely more on a response strategy than on a place strategy, consistent with an abnormal emphasis on idiothetic information. It is possible that mild disorientation before entering the environment might further accentuate a bias of aged rats toward using idiothetic information. Reliance on self-motion information could cause spatial representations of aged rats to remain rigid despite changes in the external environment, to fail to rotate with landmarks, or to delay their switch from dependence on internal to external cues (Rosenzweig et al., 2003).

Second, aged rats may have impaired vision, which would encourage them to rely more on other information, such as idiothetic cues. However, place cells of aged rats in our experiment did on some occasions rotate with visual landmarks, and, as seen by Rosenzweig 
et al. (2003), they did eventually switch to firing on the basis of the visual cues. These findings suggest that rather than impaired visual acuity, aged rats may suffer impaired visual attention. Indeed, aged rats are known for poor performance on visual attention tasks (Muir et al., 1999), and fluctuations in attention to visual landmarks would account for fluctuations in place fields.

Third, on each test, aged rats may use only a subset of available cues (Tanila et al., 1997a), and sometimes their representations may fail to incorporate the salient landmark cues. Even in young rats, place fields are sometimes determined only by particular cues, such as the floor, rather than prominent cues on the walls of an environment (Jeffery and Anderson, 2003). In light of our current data, perhaps all of the available spatial information is not fully integrated into the spatial representations of aged animals or, on some occasions, they are guided mainly by cues other than the salient visual landmarks.

Fourth, aged rats may learn spatial information at a slower rate than young rats. In our experiment, aged rats required more experience to create new spatial representations and to anchor these representations to the external landmarks. These findings provide an important parallel to the well documented delay in acquisition of spatial tasks in aged animals (for review, see Rapp and Amaral, 1992; Foster, 1999). Furthermore, it has been suggested that, despite a weakened associative learning system, the aged rats could, through additional training, more often recall the correct hippocampal representation and improve water maze performance (Barnes et al., 1997; Redish and Touretzky, 1999). Our experiment provides evidence that impaired anchoring of spatial representations to visual cues does indeed relate to water maze performance and that this binding of the spatial representations to environment cues can be accomplished through additional training by aged memory-impaired rats. These results are consistent with models of hippocampal spatial representation in which new place fields for a novel environment are first created and then gradually the external cues are bound to the new representation (McNaughton et al., 1996;

Redish and Touretzky, 1997; Samsonovich and McNaughton, 1997). Because aged rats in our experiment constructed spatial representations more slowly, the two components appeared in sequence and not simultaneously as in young rats. Delayed learning alone, however, cannot account for the findings that, even in our later recording sessions, place fields of aged rats inconsistently followed the visual landmarks and that, even in a familiar environment, place fields of aged rats were slow to realign themselves with the room cues (Rosenzweig et al., 2003). These two results may explain why some
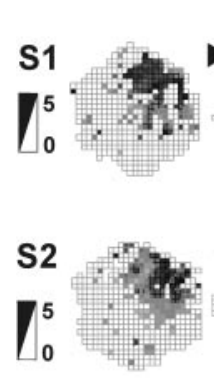

\section{S3}

$\nabla_{0}^{6}$

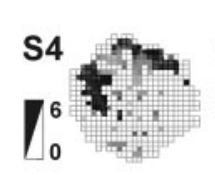

A.
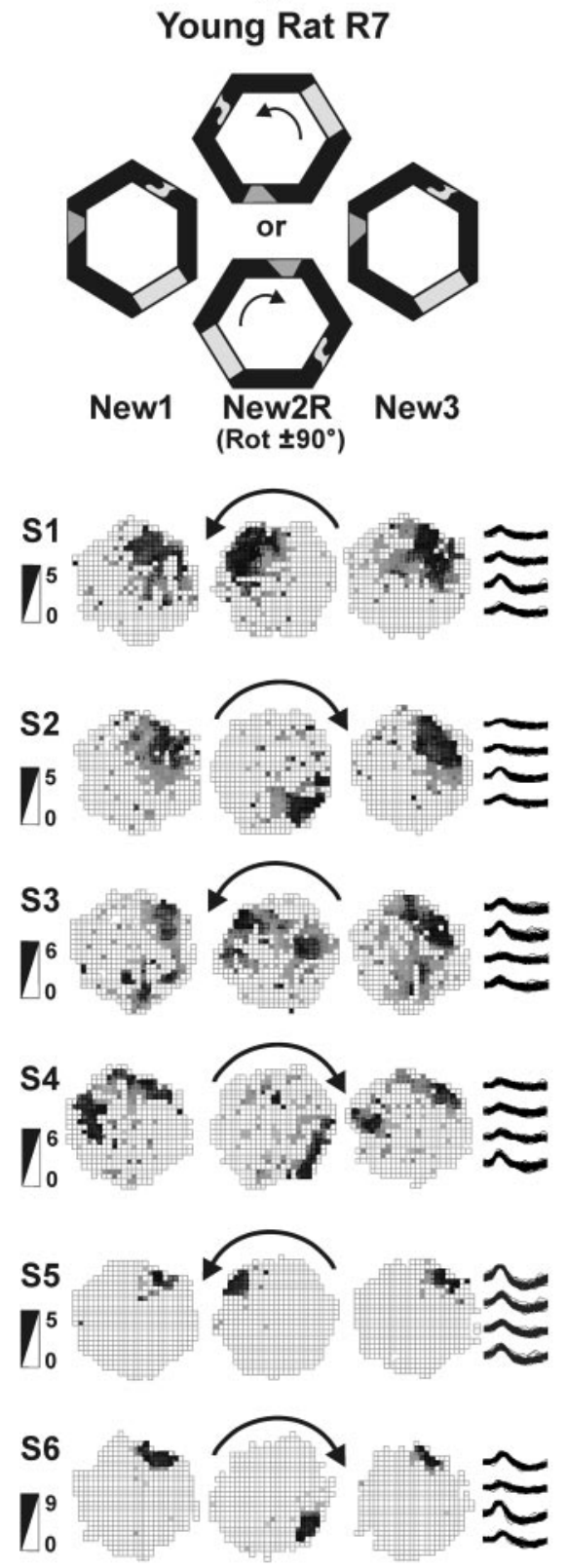

Figure 5. Place field responses of young rats to arena rotations over many sessions. Rats explored the novel environment during original and rotated orientations. The rotations alternated directions, counterclockwise in odd-numbered sessions and clockwise in even-numbered sessions (denoted by arrows). One cell from each session is shown as representative of the entire ensemble. Typically, simultaneously recorded cells responded similarly to the environmental change. Mean responses of each ensemble for each session are shown in Figure 7. Sessions are abbreviated S1-S6. Firing rate scales in spikes per second are provided on the left of the figure; example tetrode waveforms of each cell are shown on the right. $A$, Young rat R7 recorded for six sessions. The place fields rotated with the landmarks each time in trial New2R. Sessions 1 and 2 show recordings from the same cell, whereas sessions 3- 6 involve different cells. B, Young rat R9 recorded for three sessions. The place fields rotated with the landmarks each time in trial New2R. The same cell is shown across all sessions.

aged rats never reach the same level of accuracy in their spatial navigation as young rats (Gallagher et al., 1993).

\section{Age-associated neurobiological changes affecting spatial representation}

We suggest that all the manifestations of weakened external cue control arise from the same set of underlying age-associated neurobiological changes to the hippocampal circuitry. First, the number of synaptic contacts from entorhinal cortex to dentate gyrus and CA 3 is 
A.

Aged Rat R5

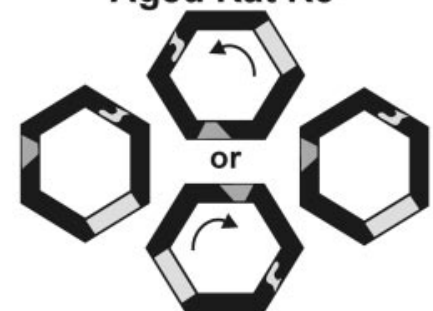

New1 New2R New3 (Rot $\pm 90^{\circ}$ )

s
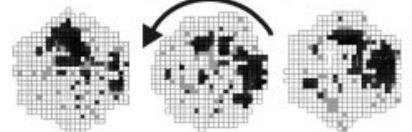

$\approx$
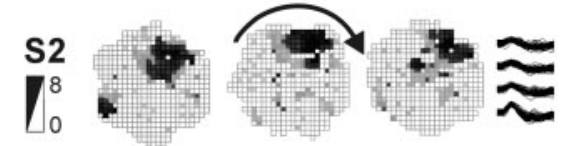

s3
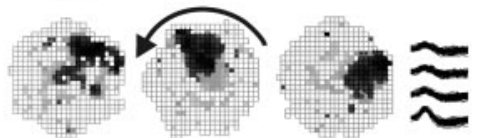

$\mathbf{S 4}$
$\left.\right|_{0} ^{7}$
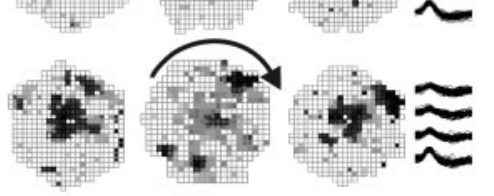

S5

$\mathbf{I}_{0}^{10}$<smiles>[InH2]</smiles>
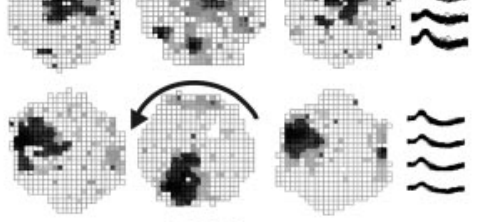

$\mathbf{S}_{0}^{6}$
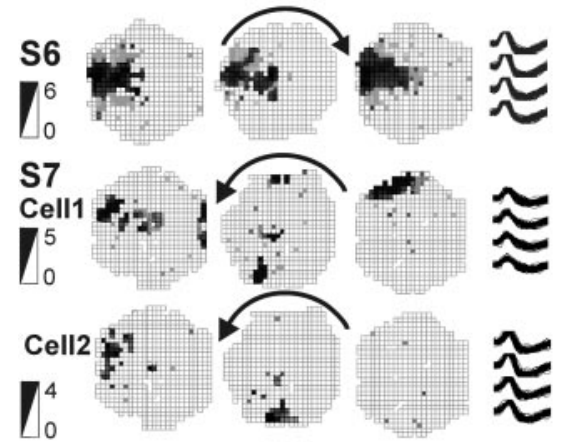

S8
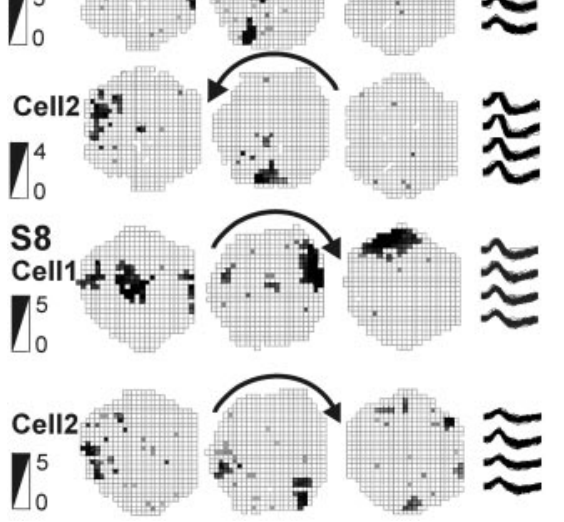

Figure 6. Place field responses of aged memory-impaired rats to arena rotations over repeated sessions. For explanations, see Figure 5. Sessions are abbreviated S1-S8. Illustrations for sessions 7 and 8 contain two cells to show remapping within the novel environment. $A$, Aged memory-impaired rat $\mathrm{R} 5$ recorded for eight sessions. The spatial representations did not rotate with the arena rotation in sessions 1 , 2, 4, and 6, but, in sessions 3 and 5, they did rotate. Sessions $2-4$ are illustrated with the same cell, whereas for all other sessions, different cells are shown. Note that even the same cell in sessions 2-4 responded differently on different days. In session 7, the place fields rotated but remapped (new location or disappeared) in trial New3 instead of returning to their original positions. In session 8, the place fields were replaced by a new one in trial New2R that rotated in trial New3 with the arena frame. Note that cell 1 (the same cell in both sessions 7 and 8) appears to have two different place fields, reflecting a kind of multi-stability. $B$, Aged memory-impaired rat R8 recorded for eight sessions. In sessions 1, 3, and 4, the spatial representations did not rotate with the arena, whereas they followed the arena rotation in sessions 2, 5, and 6. Sessions 1-6all are illustrated by the same cell, again showing that individual cells sometimes rotated and sometimes did not. Another two cells are used to illustrate both sessions 7 and 8. Note that, in sessions 7 and 8 , the two cells appear to each have two representations of the same environment without regard to the landmarks. This is strongly suggestive of multi-stability. decreased with aging, whereas the CA3 autoassociative network remains intact (Geinisman et al., 1992; Rapp et al., 1999; Smith et al., 2000). Second, the medial septum provides less cholinergic modulation to the hippocampus of aged animals (Chouinard et al., 1995; Gill and Gallagher, 1998; Sugaya et al., 1998; Nicolle et al., 1999). Third, synaptic plasticity, as measured by long-term potentiation, is weakened in aged rats (Barnes, 1994; Foster, 1999).

We propose a model of age-associated impairments in hippocampal representation that incorporates these neurobiological changes and aspects of two well known computer models of hippocampal function (Hasselmo et al., 1995; McNaughton et al., 1996). Hippocampal information processing has been modeled as an attractor network that can maintain its state despite small external disturbances but flips between states if challenged with strong enough novel sensory input (McNaughton et al., 1996; Redish and Touretzky, 1997; Samsonovich and McNaughton, 1997). During entry to an environment, such a network settles on a representation on the basis of stored and incoming sensory information. To set the stage for learning, the cholinergic input acts to focus attention and to switch the hippocampus from recalling stored information into a learning mode by altering the balance between entorhinal versus CA3 inputs to CA1 (Hasselmo et al., 1995). In young rats, even a modest change in environment or task demands is sufficient to induce the switch from recalling a stored representation of the familiar environment to creating a new representation for the changed environment. In contrast, in aged rats, the diminished entorhinal input may not transmit sufficient details of changed external sensory information to induce the switch, leaving the CA3 auto-associative network to complete the familiar pattern, resulting in the observed initial rigidity of their place cells. Decreased cholinergic modulation might result in weakened synaptic plasticity and attentional deficits, resulting in additional delay in the association of external landmarks to internal representations (Hasselmo and Bower, 1993; Leutgeb and Mizumori, 1999; Ikonen et al., 2002). Finally, entorhinal input with reduced details of external cues and impaired function of the cholinergic switching mechanism render the attractor network susceptible to inappropriate flips of its state, resulting in multi-stability of place fields.

In conclusion, weakened control of external cues over the spatial representations of aged rats manifests itself in three ways: rigidity, delayed control by external cues, 


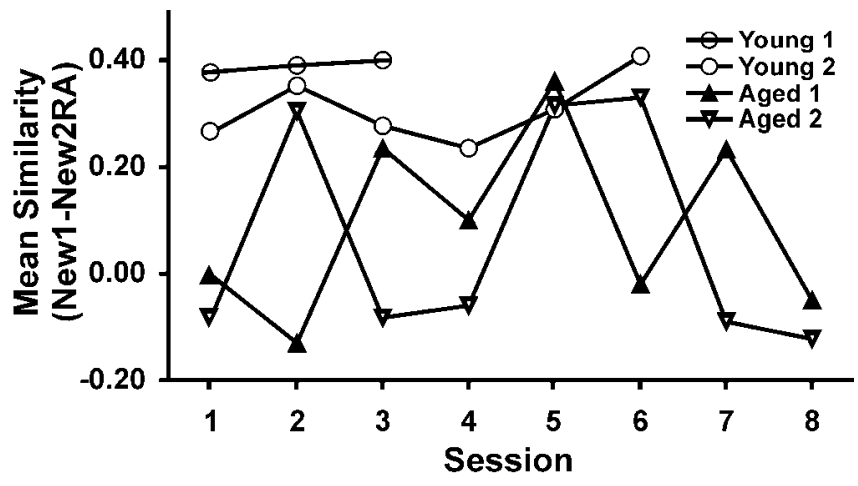

Figure 7. Reactions to rotated novel arena (aligned). The mean correlation of all place fields recorded simultaneously for each rat was calculated between the trials New1 and New2RA. Rats whose place fields did rotate with the arena have a high correlation ( $\sim 0.25$ or above). Rats whose place fields did not rotate with the arena have a low correlation ( $\sim 0$ or below). The spatial representations of young rats had high rotated correlations on every occasion. The spatial representations of aged memory-impaired rats had low correlations in the initial session but, in the second or third session, the correlations were as high as in young rats. In sessions thereafter, the place fields of the aged rats had low correlations on some occasions and high correlations on other occasions.

and multi-stability. On the basis of evidence for all three phenomena within the same experiment, we attempted to reconcile previously conflicting findings. Future studies will tighten the connections between age-associated neurobiological changes and these neurophysiological manifestations of age-associated learning impairment.

\section{References}

Barnes CA (1988) Aging and the physiology of spatial memory. Neurobiol Aging 9:563-568.

Barnes CA (1994) Normal aging: regionally specific changes in hippocampal synaptic transmission. Trends Neurosci 17:13-18.

Barnes CA, Nadel L, Honig WK (1980) Spatial memory deficit in senescent rats. Can J Psychol 34:29-39.

Barnes CA, Suster MS, Shen J, McNaughton BL (1997) Multistability of cognitive maps in the hippocampus of old rats. Nature 388:272-275.

Chouinard ML, Gallagher M, Yasuda RP, Wolfe BB, McKinney M (1995) Hippocampal muscarinic receptor function in spatial learning-impaired aged rats. Neurobiol Aging 16:955-963.

Fischer W, Chen KS, Gage FH, Bjorklund A (1992) Progressive decline in spatial learning and integrity of forebrain cholinergic neurons in rats during aging. Neurobiol Aging 13:9-23.

Foster TC (1999) Involvement of hippocampal synaptic plasticity in agerelated memory decline. Brain Res Rev 30:236-249.

Gallagher M, Burwell RD (1989) Relationship of age-related decline across several behavioral domains. Neurobiol Aging 10:691-708.

Gallagher M, Burwell R, Burchinal M (1993) Severity of spatial learning impairment in aging: development of a learning index for performance in the Morris water maze. Behav Neurosci 107:618-626.

Geinisman Y, de Toledo-Morrell L, Morrell F, Persina IS, Rossi M (1992) Age-related loss of axospinous synapses formed by two afferent systems in the rat dentate gyrus as revealed by the unbiased stereological dissector technique. Hippocampus 2:437-444.

Gill TM, Gallagher M (1998) Evaluation of muscarinic M2 receptor sites in basal forebrain and brainstem cholinergic systems of behaviorally characterized young and aged Long-Evans rats. Neurobiol Aging 19:217-225.

Hasselmo ME, Bower JM (1993) Acetylcholine and memory. Trends Neurosci 16:218-222.

Hasselmo ME, Schnell E, Barkai E (1995) Dynamics of learning and recall at excitatory recurrent synapses and cholinergic modulation in rat hippocampal region CA3. J Neurosci 15:5249-5262.

Ikonen S, McMahan R, Gallagher M, Eichenbaum H, Tanila H (2002) Cholinergic system regulation of spatial representation by the hippocampus. Hippocampus 12:386-397.
Jeffery KJ, Anderson MI (2003) Dissociation of the geometric and contextual influences on place cells. Hippocampus 13:868-872.

Leutgeb S, Mizumori SJ (1999) Excitotoxic septal lesions result in spatial memory deficits and altered flexibility of hippocampal single-unit representations. J Neurosci 19:6661-6672.

Markus EJ, Barnes CA, McNaughton BL, Gladden VL, Skaggs WE (1994) Spatial information content and reliability of hippocampal CA1 neurons: effects of visual input. Hippocampus 4:410-421.

McNaughton BL, Barnes CA, Gerrard JL, Gothard K, Jung MW, Knierim JJ, Kudrimoti H, Qin Y, Skaggs WE, Suster M, Weaver KL (1996) Deciphering the hippocampal polyglot: the hippocampus as a path integration system. J Exp Biol 199:173-185.

Mizumori SJ, Kalyani A (1997) Age and experience-dependent representational reorganization during spatial learning. Neurobiol Aging 18:651-659.

Muir JL, Fischer W, Bjorklund A (1999) Decline in visual attention and spatial memory in aged rats. Neurobiol Aging 20:605-615.

Nicolle MM, Colombo PJ, Gallagher M, McKinney M (1999) Metabotropic glutamate receptor-mediated hippocampal phosphoinositide turnover is blunted in spatial learning-impaired aged rats. J Neurosci 19:9604-9610.

O’Keefe J, Dostrovsky J (1971) The hippocampus as a spatial map. Preliminary evidence from unit activity in the freely-moving rat. Brain Res 34:171-175.

Oler JA, Markus EJ (2000) Age-related deficits in the ability to encode contextual change: a place cell analysis. Hippocampus 10:338-350.

Rapp PR (1998) Representational organization in the aged hippocampus. Hippocampus 8:432-435.

Rapp PR, Amaral DG (1992) Individual differences in the cognitive and neurobiological consequences of normal aging. Trends Neurosci 15:340-345.

Rapp PR, Stack EC, Gallagher M (1999) Morphometric studies of the aged hippocampus. I. Volumetric analysis in behaviorally characterized rats. J Comp Neurol 403:459-470.

Redish AD, Touretzky DS (1997) Cognitive maps beyond the hippocampus. Hippocampus 7:15-35.

Redish AD, Touretzky DS (1999) Separating hippocampal maps. In: The hippocampal and parietal foundation of spatial cognition (Burgess $\mathrm{N}$, Jeffery KJ, O’Keefe J, eds), pp 203-219. Oxford: Oxford UP.

Redish AD, McNaughton BL, Barnes CA (1998) Reconciling Barnes et al. (1997) and Tanila et al. (1997a,b). Hippocampus 8:438-443.

Rosenzweig ES, Redish AD, McNaughton BL, Barnes CA (2003) Hippocampal map realignment and spatial learning. Nat Neurosci 6:609-615.

Samsonovich A, McNaughton BL (1997) Path integration and cognitive mapping in a continuous attractor neural network model. J Neurosci 17:5900-5920.

Shapiro M (2001) Plasticity, hippocampal place cells, and cognitive maps. Arch Neurol 58:874-881.

Skaggs WE, McNaughton BL, Gothard KM, Markus EJ (1993) An information theoretic approach to deciphering the hippocampal code. In: Advances in neural information processing systems (Hanson SJ, Cowan JD, Giles CL, eds), pp 1030-1037. San Mateo, CA: Morgan Kaufmann.

Smith TD, Adams MM, Gallagher M, Morrison JH, Rapp PR (2000) Circuitspecific alterations in hippocampal synaptophysin immunoreactivity predict spatial learning impairment in aged rats. J Neurosci 20:6587-6593.

Sugaya K, Greene R, Personett D, Robbins M, Kent C, Bryan D, Skiba E, Gallagher M, McKinney M (1998) Septo-hippocampal cholinergic and neurotrophin markers in age-induced cognitive decline. Neurobiol Aging 19:351-361.

Tanila H, Shapiro M, Gallagher M, Eichenbaum H (1997a) Brain aging: changes in the nature of information coding by the hippocampus. J Neurosci 17:5155-5166.

Tanila H, Sipila P, Shapiro M, Eichenbaum H (1997b) Brain aging: impaired coding of novel environmental cues. J Neurosci 17:5167-5174.

Uttl B, Graf P (1993) Episodic spatial memory in adulthood. Psychol Aging 8:257-273.

White NM, McDonald RJ (2002) Multiple parallel memory systems in the brain of the rat. Neurobiol Learn Mem 77:125-184.

Wilson IA, Ikonen S, McMahan RW, Gallagher M, Eichenbaum H, Tanila H (2003) Place cell rigidity correlates with impaired spatial learning in aged rats. Neurobiol Aging 24:297-305.

Wilson MA, McNaughton BL (1993) Dynamics of the hippocampal ensemble code for space. Science [Erratum (1994) 264:16] 261:1055-1058.

Wood ER, Dudchenko PA (2003) Aging, spatial behavior and the cognitive map. Nat Neurosci 6:546-548. 